\title{
Retrospective Dosimetric Analysis of Bone Marrow Sparing vs Non Bone Marrow Sparing Image Guided Volumetric Modulated Arc Therapy in Intact Carcinoma Cervix Patients
}

\section{Kiran Chigurupalli^,†,1, Apoorv Vashistha ${ }^{2}$, Deepanjali Patel ${ }^{3}$, Ramesh Purohit ${ }^{4}$ Shalu Peter ${ }^{5}$, Menal Bhandari ${ }^{6}$}

\footnotetext{
${ }^{1}$ Assistant Professor ,M.D Radio Therapy, Affiated To Geetnajali University \& Geetanjali Cancer Center

${ }^{2}$ Medical Physicist, M.Sc Physics, Affiliated To Geetnajali University 8 Geetanjali Cancer Center - Co- Author

${ }^{3}$ Junior Resident , M.B.B.S, Affiliated To Geetnajali University 83 Geetanjali Cancer Center

${ }^{4}$ Assistant Professor, M.D Radio Therapy, Affiliated To Geetnajali University Es Geetanjali Cancer Center

${ }^{5}$ Medical Physicist, M.Sc Radiation Physics, Affiliated To Geetnajali University \& Geetanjali Cancer Center

${ }^{6}$ Assistant Professor, M.D Radio Therapy, Affiliated To Geetnajali University \& Geetanjali Cancer Center
}

(ODOI: https://doi.org/10.15520/jcmro.v2i10.214

Accepted 08-10-2019; Received 15-09-2019; Publish Online 11-10-2019

Reviewed By: Dr. Sorush Niknamian Department: Reviewer/CMRO

\begin{abstract}
Aim: Aim of this study was to compare dosimetric parameters of bone marrow (BM) and non bone marrow sparing with state of the art technique image guided volumetric modulated arc therapy in the treatment of Carcinoma Cervix.

Methods and Materials: The retrospective Dosimetric study was conducted on 10 consecutive patients of biopsy-proven invasive cervical cancer attending the outpatient department of Geetanjali cancer centre. For treatment planning, patients were scanned on a GE Optima-520, 16 slice fan-beam CT (FBCT) scanner with $2.5 \mathrm{~mm}$ slice thickness in the supine position on a customized vacuum cushion with simulation tattoos and alignment lasers. All the simulation scans were taken after following a bladder and bowel preparation protocol, aimed at an empty rectum and full bladder, starting prior to the initial planning scan and continuing throughout the treatment. The prescribed dose to PTV was 50Gy in 25 fractions (2Gy/fraction) and from second week of radiotherapy $50 \mathrm{mg} / \mathrm{m}^{2} /$ week cisplatin chemotherapy was administered for five weeks. Two sets of plans were generated for all the 10 patients using Volumetric Arc Radiotherapy (VMAT) in Monaco v 5.11.02(Elekta, Crawley, UK) treatment planning system. Keeping all constraints in mind planning optimization was performed with no compromise in coverage of PTV.

Results: In the present study V5, V10, V20, V30 were all significantly lower in BMS-IG/VMAT plan. Maximum significance was noted at V20. Value of V40 is lower in BM-IG/VMAT plan, but statistically not significant.

Conclusion: Treatment of carcinoma cervix is restricted as a result of toxicity of the surrounding normal structures and HT. HT could be significantly reduced by bone marrow sparing, which could be aided by IGRT/VMAT. This modality could allow completion of treatment with prescribed dose and concurrent chemotherapy in allotted stipulated time. More prospective randomized control trials are needed, to prove its efficacy.
\end{abstract}

Key words: Bone Marrow Sparing-Hematological Toxicity-Image Guidance/Volumetric Modulated Arc Therapy 


\section{Retrospective Dosimetric Analysis of Bone Marrow Sparing vs Non Bone Marrow Sparing Image Guided Volumetric Modulated Arc Therapy in Intact Carcinoma Cervix Patients

\section{INTRODUCTION:}

Prevalence of Cervical cancer is high in developing nations and it is estimated that close to 569,847 new cases and 311.356 deaths, related to carcinoma cervix were noted in 2018 [1]. Concurrent chemo-radiotherapy is used as a standard treatment protocol. Concurrent chemo-radiotherapy has significantly improved tumor control, overall survival and progression free survival [2]. Many studies have reported that when the planned dose is delivered to the tumor using conventional techniques such as, 2D and 3DCRT, a larger volume of bone marrow is irradiated and higher dose is delivered to the exposed bone marrow[[3, 4]. More than $50 \%$ of active bone marrow in adults is located in the pelvic and neighboring bones, which, when irradiated during the pelvic radiotherapy leads to decrease in the level of blood counts [3] . Concurrent chemo-radiotherapy to pelvis increases the hematological toxicity (HT) particularly leucopenia, neutropenia and thrombocytopenia. This Hematological Toxicity can cause uncalled interruption in the radiotherapy schedule or reduction in the no. of chemotherapy cycles scheduled. The fore mentioned problems results in reduced tumor control and accelerated tumor progression [5]. As a result, sparing bone marrow as much as possible has become a priority in delivering precision RT [6]. Using IMRT/VMAT radiation doses can be delivered with high conformality, while avoiding nearby critical structures. The benefits of IMRT/VMAT were documented in head and neck cancer $[7,8]$, prostate cancer $[3,9]$. The results of RTOG 0418 phase II trial shown hematological toxicities can be associated with mean dose and higher dose irradiation volume of BM [10] [10].Few studies associated hematological toxicity with $\mathrm{V}_{10 G y} \%$ and $\mathrm{V}_{20 G y} \%[11,12]$. The usage of image guided volumetric modulated arc therapy technique (IG/VMAT) is on raise in recent times and its role is well established [13].Aim of the present study is to investigate Dose Volume Histogram parameters between state of the art technique IGRT/VMAT plan with and without bone marrow sparing and compare the results.

\section{MATERIALS AND METHODS:}

The present study is a retrospective Dosimetric study conducted on 10 consecutive patients of biopsy-proven invasive cervical cancer attending the outpatient department of Geetanjali cancer centre, Udaipur, Rajasthan, India between January 2019 and April 2019. As per FIGO [14] two patients were IIB, two patients were IIIA, six patients were IIIB. For treatment planning, patients were scanned on a GE Optima520, 16 slice fan-beam CT (FBCT) scanner with $2.5 \mathrm{~mm}$ slice thickness in the supine position on a customized vacuum cushion with simulation tattoos and alignment lasers. All the simulation scans were taken after following a bladder and bowel preparation protocol, aimed at an empty rectum and full bladder, starting prior to the initial planning

* Corresponding author.

† Email: kiran.doc2010@gmail.com scan and continuing throughout the treatment. The bowel preparation consists of a low bulk diet, and rectum voiding before treatment. The bladder preparation requires 1 litre of water to be consumed $30 \mathrm{~min}$ before planning scan and every treatment time. Fludicial markers were inserted into the vaginal cuff to visualize it on $\mathrm{CT}$ images. $\mathrm{CT}$ images were obtained from T12 to middle third of femur. Oral and intravenous contrast were administered to all the patients before acquiring CT images.

Gross Tumor volume (GTV) defined as gross tumor, its extent and positive lymph nodes as seen on the image. CTV was defined, based on the primary tumor extent and positive node involved with an additional margin for including microscopic spread. PTV was created from the CTV with an additional margin of 5-7 $\mathrm{mm}$ in all direction. Based on standard Radiation Therapy Oncology Group guidelines (RTOG) [15-17]. Organ at Risk included bladder, rectum, both femoral head, small bowel and the bone marrow . The low-density regions inside the bone were contoured by free hand as the surrogate for BM as demonstrated by Mahanshetty et al [18]. To bring uniformity the widow was adjusted to bone range while contouring the surrogate for bone marrow. All contouring was done by a single physician and verified by another physician for all plans, so that inter-observer variations could be eliminated. The cranial border of bone marrow contour began at $1 \mathrm{~cm}$ above the uppermost border of planning target volume (PTV) and ended at $1 \mathrm{~cm}$ below the lower border of the PTV. The rectum was outlined up to sigmoid flexure. The small bowel included the entire peritoneal cavity (not individual loops of bowel) up to L3. The prescribed dose to PTV was 50Gy in 25 fractions (2Gy/fraction) and from second week of radiotherapy $50 \mathrm{mg} / \mathrm{m}^{2} /$ week cisplatin chemotherapy was administered for five weeks.

Two sets of plans were generated for all the 10 patients using Volumetric Arc Radiotherapy (VMAT) in Monaco v 5.11.02(Elekta, Crawley, UK) treatment planning system , using Monte Carlo Algorithm using two full arcs.3mm grid space along with $5 \mathrm{~mm}$ minimum segment width was used for all the plans. Keeping all constraints in mind planning optimization was performed with no compromise in coverage of PTV.

V95 of PTV received $\geq 95 \%$ of the prescribed dose in both Bone Marrow Sparing and Normal VMAT plan. Constraints for bowel bag $\mathrm{V} 40<30 \%$, for rectum and bladder $\mathrm{V} 40<50-60 \%$ and for both femoral head V30 $<20 \%$. Constraints to bone marrow were V10 $<90 \%$ and V $20<75 \%$ of prescribed dose.

Before treatment delivery CBCT scans were acquired in Elekta XVI with software version 5.0.2, using large volume scan protocol mode settings $120 \mathrm{kV}, 80 \mathrm{~mA}, 28 \mathrm{mAs}$ and acquisition angle range 360, maximum reconstruction diameter $40 \mathrm{~cm}$, using the half-fan bowtie filter for Image Guided Radiotherapy during 25 fraction treatment course. Positional error, if any, were corrected and the prescribed dose was delivered.

Dosimetric comparisons of plans were done based on the following parameters extracted from dose volume histogram 
(DVH): homogeneity index (HI), conformity index (CI), V5, V10, V20, V30, V40 of pelvic bone marrow,V10, V20, V30, V40 of small bowel, V20, V30,V40 of bladder and V10, V20, V30, V40 of rectum. The HI was used to analyze the dose uniformity and defined as D5/D95. The lower the HI, the better the dose homogeneity. $\mathrm{CI}=$ the percentage of the PTV volume receiving at least 50 Gy $\times$ the ratio of the volume of the PTV receiving at least 50 Gy to the total 50 Gy volume. The closer the CI value was to 1 , the better the dose conformity [19].

SPSS software was used in analyzing the data (version 20.0,SPSS Inc, USA). Data was expressed in the form of mean \pm standard deviation $(\chi \pm \mathrm{s})$. Paired two-tailed students $t$ test was used to evaluate the significance of differences.

\section{RESULTS:}

Homogenisity index and conformity index of the PTV coverage

The values of HI and CI were mentioned in Table 1 . the results revealed no statistical difference between $\mathrm{HI}$ and $\mathrm{CI}$ with bone marrowsparing and normal IG/VMAT

Comparision of dosimetric parameters of OARs for two modalities

Various doismetric parameters of bone marrow, small bowel, bladder, and rectum were described in theTable 2 . Statistical difference was seen $\mathrm{V}_{5}, \mathrm{~V}_{10}, \mathrm{~V}_{20}, \mathrm{~V}_{30}$ pelvic bone marrow $(P=0.006, P=0.001, P=0.000, P=0.003)$. Irradiated Bone marrow volume was significantly reduced in both low and high dose areas by BMS-IG/VMAT technique. $\mathrm{V}_{40}$ of bone marrow was not significant. No significance was noted in volume parameters of small bowel and bladder. For rectum, $\mathrm{V}_{20}$ demonstrated notable significance between BM-IG/VMAT and IG/VMAT. The most possible explanation for the results in small bowel, bladder and rectum might be due to increase in irradiated volume of small bowel, bladder, rectum to achieve lower irradiated volume of pelvic bone marrow.
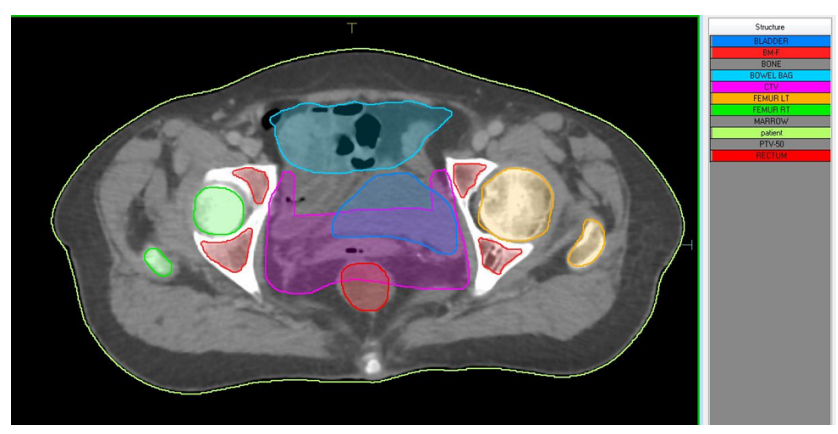

Figure 1. Contured structure set

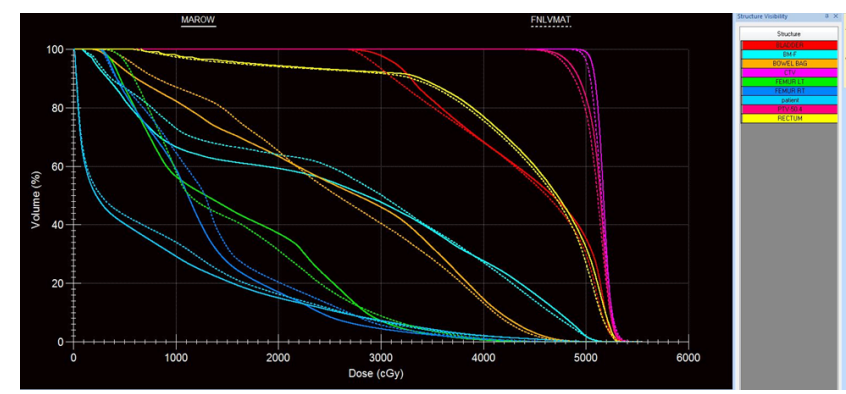

Figure 2. comparison of DVH of BMS-IG/VMAT and IG/VMAT

\section{DISCUSSION:}

The purpose of our study was to compare bone marrow sparing IG/VMAT with normal IG/VMAT planning ,to reduce volume of irradiated pelvic bone marrow , which could be an important contributing factor for acute bone marrow toxicity $[3,12,15]$.Concurrent chemotherapy with highly conformal radiotherapy has improved the survival rates, as well as increasing hematological toxicity [20]. Prolonged treatment duration due to interruption caused by $\geq$ grade 2 hematological toxicity, will have negative impact on overall survival and also local control [21-23]. Results of Rose BS et al., showed that grade 3 neutropenia is high, if the volume of $\mathrm{PBM}$ (pelvic bone marrow) receiving $10 \mathrm{~Gy}$ is $>95 \%$ than in those patients in whom $<95 \%$ of the PBM received this dose $(63.8 \%$ vs $24.6 \%$; p <0.001) [24]. Albuquerque K reported that grade $\geq 2 \mathrm{HT}$ can be strongly predicted by volume of PBM receiving 20 Gy. The risk of HT increased by a factor of 4.5 when $>80 \%$ of PBM received 20 Gy $[15,25]$. In the present study V5, V10, V20, V30 were all significantly lower in BMS-IG/VMAT plan . Maximum significance was noted at $\mathrm{V}_{20}$. Value of V40 is lower in BM-IG/VMAT plan, but statistically not significant. RTOG 0418 trial described that $\geq$ grade $2 \mathrm{HT}$ is more if the volume of $\mathrm{PBM}$ receiving 40 Gy is $>37 \%(p=0.04)$ [10]. Although the constraints were not achieved in the present study, the mean values of all volumes were significantly less. This could be due to not including femoral heads in the bone marrow; as a result the absolute value of pelvic bone marrow is decreased. Rest of the studies included femoral heads in the volume of pelvic bone marrow. Other possible reason could be that we conducted this dosimetric analysis on intact cervical patients wherein generous PTV was considered. All our patients received 50 Gy in 25 fractions and rest of the studies $45 \mathrm{~Gy}$ in 25 fractions was delivered. Major limitation of our study was that the constraints given to the bladder and rectum (V40 $\leq 40-60 \%)$ were not met. As majority of rectum and bladder volume was lying within the PTV. RTOG 0418 [10] [10] described in patients of endometrial carcinoma the constraints given to bladder and rectum of V45 $\leq 35 \%$ and V45 $\leq 60 \%$ were achieved in only $33.3 \%$ and $22.8 \%$ respectively. By analyzing the current data, we concluded that the constraints given were unrealistic. RTOG 0418 study suggested that new trials should try to liberalize the dose constraints to a certain level [10]. Study by Mell LK et al., BMS-IMRT 


\section{Retrospective Dosimetric Analysis of Bone Marrow Sparing vs Non Bone Marrow Sparing Image Guided Volumetric Modulated Arc Therapy in Intact Carcinoma Cervix Patients

Table 1.

\begin{tabular}{lllll}
\hline & BMS- & IG/VMAT & t-VALUE & P -VALUE \\
& IG/VMAT & & & \\
HI & $1.0697 \pm 0.02737$ & $1.0685 \pm 0.02232$ & -0.377 & 0.715 \\
CI & $0.9765 \pm 0.02289$ & $0.9733 \pm 0.02354$ & -1.316 & 0.221 \\
\hline
\end{tabular}

Table 2.

\begin{tabular}{llllll}
\hline OAR & DOSIMETRIC PARAMETERS & BMS-IG/VMAT & IG/VMAT & t value & P value \\
BM & V5 & $91.2280 \pm 7.13428$ & $93.6880 \pm 6.08795$ & 3.604 & .006 \\
& V10 & $78.1100 \pm 8.08528$ & $85.4230 \pm 8.20678$ & 4.618 & .001 \\
& V20 & $65.9200 \pm 7.12604$ & $72.3970 \pm 7.27631$ & 5.923 & .000 \\
& V30 & $53.1480 \pm 7.26403$ & $57.5060 \pm 7.13710$ & 4.063 & .003 \\
\multirow{5}{*}{ SMALL BOWEL } & V40 & $35.5680 \pm 7.79528$ & $37.1360 \pm 7.77452$ & 1.760 & .112 \\
& V10 & $86.9220 \pm 8.52063$ & $88.2370 \pm 7.65616$ & 1.0834 & .100 \\
& V20 & $63.8630 \pm 9.51192$ & $65.0630 \pm 8.63531$ & 0.55 & .596 \\
BLADDER & V30 & $40.6630 \pm 11.71018$ & $38.5870 \pm 11.78525$ & -1.147 & .281 \\
& V40 & $17.9270 \pm 7.58068$ & $16.4410 \pm 7.31540$ & -1.328 & .217 \\
& V20 & $99.1430 \pm 1.75260$ & $99.8800 \pm 0.28079$ & 1.283 & .232 \\
RECTUM & V30 & $93.3480 \pm 7.66451$ & $92.6870 \pm 6.01752$ & -0.752 & .471 \\
& V40 & $75.1530 \pm 12.74223$ & $74.5400 \pm 12.13383$ & -1.385 & .199 \\
& V10 & $97.2090 \pm 4.73948$ & $97.0530 \pm 4.95039$ & -1.477 & .174 \\
& V20 & $95.9330 \pm 6.12140$ & $95.8050 \pm 6.2594$ & -2.437 & .038 \\
& V30 & $94.0340 \pm 7.12564$ & $93.8030 \pm 7.03076$ & -0.887 & .398 \\
& V40 & $82.9750 \pm 10.19145$ & $83.0550 \pm 9.89506$ & 0.151 & .883 \\
\hline
\end{tabular}

with conventional radiotherapy techniques in seven intact cervical cancer patients revealed that, V40 of bladder and rectum was $73.6 \%$ and $83.7 \%$ respectively [10].

Another unclear issue is, to what extent the degree of sparing is necessary for significant reduction in toxicity. Limiting low-dose radiation to normal tissue is difficult with conventional planning margins and current algorithms even after using modern modalities like IGRT/VMAT technique. it would be advisable to reduce the planning margins to improve bone marrow-sparing using these techniques. organ motion is a significant problem, which needs to be better understood and also quantified. study By Krishnan et al , showed the sparing of bone marrow with VMAT at different dose and volume level was much better than IMRT In other words, IGRT/VMAT technique could be used as a better tool to permit safe reduction in the planning margins that could improve bone marrow-sparing

\section{CONCLUSION:}

Treatment of carcinoma cervix is restricted as a result of toxicity of the surrounding normal structures and HT. HT could be significantly reduced by bone marrow sparing, which could be aided by IGRT/VMAT. This modality could allow completion of treatment with prescribed dose and concurrent chemotherapy in allotted stipulated time. More prospective randomized control trials are needed, to prove its efficacy.

\section{REFERENCES}

[1] Global cancer statistics 2018: GLOBOCAN estimates of incidence and mortality worldwide for 36 cancers in 185 countries [2] Green JA Kirwan JM Tierney JF et al. Survival and recurrence after concomitant chemotherapy and radiotherapy for cancer of the uterine cervix: a systematic review and meta-analysis. Lancet. 2001;358:781-786.
[3] Roeske JCKJ. Dosimetric predictors of acute hematologic toxicity in cervical cancer patients treated with concurrent cisplatin and intensity-modulated pelvic radiotherapy. Int J Radiat Oncol Biol Phys. 2006;66:1356-1365.

[4] Reducing uncertainties about the effects of chemoradiotherapy for cervical cancer: a systematic review and metaanalysis of individual patient data from 18 randomized trials. J Clin Oncol. 2008;26:5802-5812.

[5] Treatment resistance of solid tumors: role of hypoxia and anemia. P Thews O Hoeckel M. 2001;18:243-259.

[6] Lk, Tiryaki H, Ahn KH. Dosimetric comparison of bone marrow-sparing intensity-modulated radiotherapy versus conventional techniques for treatment of cervical cancer. Int J Radiat Oncol Biol Phys. 2008;71:1504-1510.

[7] Graff I, Serre P, Boisselier A, Bensadoun P, Ortholan RJ, C. Intensity-modulated radiotherapy in head and neck cancer: Results of the prospective study GORTEC 2004-03. Radiotherapy and Oncology. 2012;103(1):57-62.

[8] Nutting CM, Morden JP, Harrington KJ, Urbano TG, Bhide SA, Clark C; 2011.

[9] Zelefsky MJ, Kollmeier M, Cox B, Fidaleo A, Sperling D, Pei $\mathrm{X}$. Improved clinical outcomes with high-dose image-guided radiotherapy compared with non-IGRT for the treatment of clinically localized prostate cancer. Int J Radiat Oncol Biol Phys. 2012;84(1):125-154.

[10] Klopp AH, Moughan, Portelance L. Hematologic toxicity in RTOG 0418: a phase 2 study of postoperative IMRT for gynecologic cancer. Int J Radiat Oncol Biol Phys. 2013;86:8390.

[11] Fargeot PLCFP. Results of a European Organization for Research and Treatment of Cancer/Early Clinical Studies Group phase II trial of first-line irinotecan in patients with advanced or recurrent squamous cell carcinoma of the cervix. J Clin Oncol. 1999;17:3136-3142.

[12] Mell LK, Schomas, Salama JK. Association between bone marrow dosimetric parameters and acute hematologic toxicity in anal cancer patients treated with concurrent 
chemotherapy and intensity-modulated radiotherapy. Int J Radiat Oncol Biol Phys. 2008;70:1431-1437.

[13] Clinical Outcomes, Patterns of Failure, and Acute Haematologic Toxicity of Image-Guided Volumetric Modulated Arc Therapy (IG-VMAT) in the Definitive Treatment of Locally Advanced Carcinoma Cervix. Clinical Medicine Insights Oncology. 2018;12:1-10

[14] FIGO staging for carcinoma of the vulva, cervix, and corpus uteri. Committee on Gynecologic Oncology. 2014;125:97-98.

[15] Albuquerque K, Morrison CGD. Radiation-related predictors of hematologic toxicity after concurrent chemoradiation for cervical cancer and implications for bone marrowYsparing pelvic IMRT. Int J Radiat Oncol Biol Phys. 2011;79:1043-1047.

[16] Small W, Mell LK, Anderson P, Creutzberg CDL, Santos, Gaffney D. Consensus guidelines for delineation of clinical target volume for intensity-modulated pelvic radiotherapy in postoperative treatment of endometrial and cervical cancer. Int J Radiat Oncol Biol Phys. 2008;71:428-462.

[17] Lim K, Small, Portelance L, Creutzberg CJSI, Mundt A. Consensus guidelines for delineation of clinical target volume for intensity-modulated pelvic radiotherapy for the definitive treatment of cervix cancer. Int $\mathrm{J}$ Radiat Oncol Biol Phys. 2011;79:348-55.

[18] Mahantshetty U, Krishnatry R, Chaudhari S, Kanaujia A, Engineer R, Chopra S, et al. Comparison of 2 contouring methods of bone marrow on CT and correlation with hematological toxicities in non-bone marrow-sparing pelvic intensity-modulated radiotherapy with concurrent cisplatin for cervical cancer. Int J Gynecol Cancer. 2012;22:14271434.

[19] Weiss E, Siebers JV, Keall PJ. An analysis of 6-MV versus 18-MV photon energy plans for intensity-modulated radiation therapy (IMRT) of lung cancer. Radiother Oncol. 2007;82:55-62.

[20] Rose PG, Bundy BN, Watkins EB, Thigpen JT, Deppe G, Maiman MA. Concurrent cisplatin-based radiotherapy and chemotherapy for locally advanced cervical cancer. N Engl J Med. 1999;340(15):1144-53.

[21] Parker K, Gallop-Evans E, Hanna L, Adams M. Five years' experience treating locally advanced cervical cancer with concurrent chemoradiotherapy and high-dose-rate brachytherapy: results from a single institution. Int J Radiat Oncol Biol Phys. 2009;74(1):140-186.

[22] Lanciano RM, Pajak TF, Martz K, Hanks GE. The influence of treatment time on an outcome for squamous cell cancer of the uterine cervix treated with radiation: A patterns-of-care study. Int J Radiat Oncol Biol Phys. 1993;25(3):391-97.

[23] Perez CA, Grigsby PW, Castro-Vita H, Lockett MA. Carcinoma of the uterine cervix. I. Impact of prolongation of overall treatment time and timing of brachytherapy on the outcome of radiation therapy. Int J Radiat Oncol Biol Phys. 1995;32(5):1275-88.

[24] Rose, Bs, Aydogan B, Liang Y, Yeginer M, Hasselle MD, et al. Normal tissue complication probability modeling of acute hematologic toxicity in cervical cancer patients treated with chemoradiotherapy. Int J Radiat Oncol Biol Phys. 2011;79(3):800-807.

[25] Shetty SKJ, Rao. Dosimetric Advantage of VMAT Technique in Bone Marrow Sparing Than IMRT in Treatment of Cervical Cancer. International Journal of Health Sciences \& Research. 2017;(7):102-111. 